\title{
Capítulo 1 \\ Consideraciones sobre la investigación en las ciencias militares aeronáuticas en Colombia
}

\author{
Diana Paola Brochero Barragán* \\ Nora Patricia Gutiérrez Rodríguez** \\ Erika Juliana Estrada Villa***
}

* Magíster en Ciencias Militares Aeronáuticas, Escuela de Postgrados Fuerza Aérea Colombiana. Correos electrónicos: diana.brochero@fac.mil.co; diana.brochero@hotmail.com

** Jefe de programa, Maestría en Ciencias Militares Aeronáuticas, Escuela de Postgrados Fuerza Aérea Colombiana. Correo electrónico: nora.gutierrez@epfac.edu.co

*** Docente, Maestría en Ciencias Militares Aeronáuticas, Escuela de Postgrados Fuerza Aérea Colombiana. Correo electrónico: erika.estrada@epfac.edu.co 


\section{CÓMO CITAR}

Brochero, D., Gutiérrez, N., \& Estrada, E. (2021). Consideraciones sobre la investigación en las ciencias militares aeronáuticas en Colombia. En R. Mezú (Ed.), Gaviotas de luces. Un aporte desde la investigación formativa a las ciencias militares aeronáuticas en Colombia (pp. 17-32). Escuela de Postgrados Fuerza Aérea Colombiana.

\section{Colección Ciencia y Poder Aéreo N. ${ }^{0} 17$}

GAVIOTAS DE LUCES

Un aporte desde la investigación formativa

a las ciencias militares aeronáuticas en Colombia

\section{CAPÍTULO 1.}

Consideraciones sobre la investigación en las ciencias militares aeronáuticas en Colombia

ISBN: $978-958-53696-0-3$ E-ISBN: $978-958-53696-1-0$

https://doi.org/10.18667/9789585369603.01 Bogotá, Colombia

Noviembre, 2021

\section{RESUMEN}

Este capítulo presenta los resultados de un análisis sobre la trayectoria investigativa en el campo de conocimiento de las ciencias militares aeronáuticas en Colombia. Este estudio es necesario debido a que en la actualidad hay un interés por las investigaciones que transversalizan con las ciencias militares aeronáuticas marcando una tendencia en las investigaciones a nivel postgradual. De esta forma surge el interés por evidenciar la naturaleza multidisciplinaria de las ciencias militares aeronáuticas como un conjunto de disciplinas donde confluyen las teorías sociales, la ciencia, la tecnología y la doctrina militar. Con este fin se diseñó una investigación cualitativa-documental que tipifica los estudios realizados desde junio del 2016 hasta julio del 2020, la cual permitió reconocer los horizontes investigativos que involucran las líneas de investigación con características propias del ámbito militar aeronáutico durante un periodo de cuatro años.

\section{PALABRAS CLAVE}

Investigación; ciencias militares aeronáuticas; doctrina; educación militar. 
— ste capítulo es resultado del trabajo de grado denominado "Consideraciones sobre la investigación en el campo de las ciencias militares aeronáuticas" del programa de Maestría en Ciencias Militares Aeronáuticas (MACMA), el cual está enmarcado en la línea de investigación de "doctrina militar aeroespacial" dentro del eje temático "estudios en doctrina militar aeronáutica". El trabajo se formuló con el objeto de identificar las líneas de investigación de las ciencias militares aeronáuticas que fortalezcan la producción académica y la investigación dentro del programa MACMA de la Escuela de Postgrados Fuerza Aérea Colombiana (EPFAC). Para avanzar en su desarrollo, una de las tareas consistió en conocer la trayectoria investigativa de la MACMA a través de la tipificación por eje temático, objeto de la investigación y tipo de investigación. Se analizaron los trabajos de grado presentados por los maestrandos desde enero del 2016 hasta junio del 2020, identificando las fortalezas y debilidades de los procesos académicos e investigativos al interior del programa.

Este capítulo está compuesto por una descripción del contexto, el cual está enmarcado en la normatividad de la educación superior en Colombia. Primero, se presenta una reflexión introductoria y descriptiva sobre el desarrollo de la investigación formativa en las ciencias militares aeronáuticas en Colombia. Después, se muestran los principales hallazgos obtenidos del análisis de los trabajos de grado explorados de MACMA y, por último, se identifica la necesidad de determinar un horizonte investigativo.

\section{Contexto}

En el ámbito nacional las escuelas de formación de la Fuerza Aérea Colombiana son las únicas instituciones de educación superior que cuentan con programas aprobados por el Ministerio de Educación Nacional (MEN) en pregrado y postgrado en el área de las ciencias militares aeronáuticas, concebidas, área de estudio concebida como una ciencia multidisciplinaria y transdisciplinaria de los campos del conocimiento militar y aeronáutico que permiten conducir operaciones aéreas, de inteligencia, logísticas aeronáuticas, seguridad, defensa de bases aéreas y procesos administrativos.

En el año 2014 la EPFAC da apertura a la MACMA, la cual contempla en su fundamentación teórica los estudios militares y la doctrina militar aérea. A la fecha de publicación de este libro, la maestría cuenta con siete cohortes 
con un total de 142 estudiantes y 99 graduados (figura 1) de diferentes especialidades, oficiales de los cuerpos de vuelo, de seguridad y defensa de bases aérea, logístico y administrativo. En el año 2016 se contó con la primera promoción de egresados de MACMA y por ende con la presentación de los primeros trabajos de grado, requisito para alcanzar el título de magíster (Reglamento Académico, 2014).

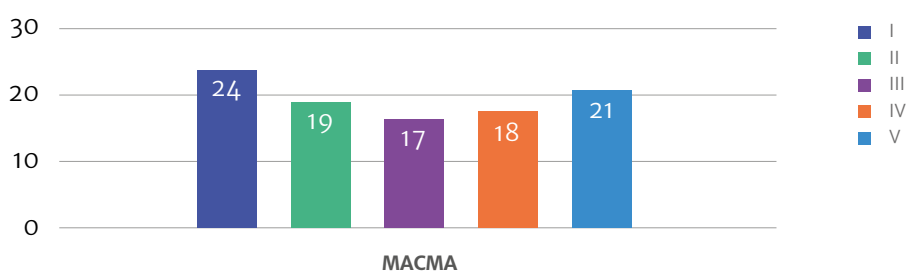

Figura 1. Graduados de la Maestría en Ciencias Militares Aeronáuticas (MACMA) por cohorte Fuente: elaboración propia.

Dicho lo anterior y con el propósito de indagar en el desarrollo investigativo, se planteó caracterizar los proyectos de grado de MACMA con un enfoque interpretativo, ajustado a los diseños cualitativos, "el cual es fundamental para los procesos de análisis e interpretación de la información" (Gómez et al., 2015, p. 230). Cabe mencionar que en el estado del arte no se encontraron informes o documentos escritos relacionados o similares al objeto de estudio de esta investigación, teniendo en cuenta el carácter exclusivo y la oferta cerrada de este programa (EPFAC, 2019).

En esa línea, los datos arrojados en las categorías contribuyen al fortalecimiento de las líneas de investigación al interior del programa MACMA, y para la toma de decisiones de la EPFAC en lo relacionado con la investigación formativa del programa. Lo anterior, teniendo en cuenta que luego de los resultados de la autoevaluación para la MACMA en el 2018, la condición denominada "investigación" arrojó una valoración poco favorable, quedando varios ítems por cumplir el nivel satisfactorio (Escuela de Postgrados FAC, 2018, p. 53).

A partir de los resultados de la autoevaluación en el 2018, se planteó conocer de cerca la gestión y el desarrollo de la condición "investigación" en el nivel formativo al interior del programa, específicamente en los trabajos de grado de MACMA, desde enero del 2016 hasta julio del 2020, los cuales se desarrollaron en el marco de un proceso "riguroso, controlado y 
sistemático de recolección de datos y análisis de información fiable y válida" (Tejedor, 2000, p. 320).

Por lo anterior, según los parámetros académicos establecidos por los lineamientos para la articulación de la investigación EPFAC (2019), las políticas del escuadrón de investigación de esta Institución de Educación Superior (IES) determinan que:

los trabajos de grado se desarrollan en el marco de la investigación formativa, la cual constituye una de las actividades fundamentales dentro del Sistema de Ciencia Tecnología e Innovación de la Fuerza Aérea Colombiana, ya que a través de esta se desarrollan las habilidades investigativas en los miembros de la FAC, tanto para la gestión de Actividades de Ciencia Tecnología e Innovación como para el desarrollo mismo de los proyectos de I+D+I, tal como lo establece el manual de investigación (p. 3).

Es así como los proyectos de grado desarrollados por los estudiantes en el periodo mencionado se ejecutaron como requisito de grado y sirvieron para enriquecer el acervo bibliográfico de cada problemática planteada por sus autores. Además, teniendo en cuenta el valor de sus contenidos académicos como trabajos que permiten ser estudiados y analizados, la información que comprenden puede ser agrupada para su análisis en categorías y los resultados son empleados como insumo estadístico y literario para las ciencias militares aeronáuticas (Sabino, 1986, p. 12).

\section{Normativa}

La EPFAC acoge lo consagrado en los fundamentos de la educación superior (Ley 30,1992) en su artículo $1 .^{\circ}$ en cuanto a la definición de esta como un proceso permanente que posibilita el desarrollo de las potencialidades del ser humano de una manera integral y lo descrito de las IEs estipulado en el Capítulo IV de la misma ley.

Por tanto, es deber de los programas de pregrado y de postgrado que ofrezcan las instituciones de educación superior, actuar en los campos de acción que la ley señala y de conformidad con sus propósitos de formación (art. 8, Ley 30 de 1992).

A su vez, los programas de maestría tienen como propósito ampliar y desarrollar los conocimientos, actitudes y habilidades para la solución 
de problemas disciplinares, interdisciplinarios o profesionales y dotar a la persona de los instrumentos básicos que la habilitan como investigador. De acuerdo con la normatividad vigente, los programas podrán ser de profundización o de investigación (Decreto 1330 del 2019).

\begin{abstract}
A lo largo de este capítulo se especifica que la MACMA es un programa de maestría de profundización, la cual debe ser entendida, conforme al Decreto 1330, como el programa que propende por avanzados conocimientos, actitudes y habilidades que permiten la solución de problemas o análisis de situaciones particulares de carácter disciplinar, interdisciplinario o profesional, por medio de la asimilación o apropiación de saberes, metodologías y, según el caso, desarrollos científicos, tecnológicos, artísticos o culturales (sección 6, Decreto 1330 del 2019).
\end{abstract}

Este decreto establece que para optar al título de un programa de maestría en profundización, el estudiante podrá cumplir con lo señalado por la institución como opción de grado, bien sea mediante un trabajo de investigación aplicada que podrá ser presentado como un estudio de caso, o con un documento de análisis, en el que explore soluciones a problemas concretos o situaciones particulares (Decreto 1330 del 2019).

\title{
Consideraciones y reflexiones sobre las ciencias militares aeronáuticas
}

Según el contexto de educación superior, en lo referente a programas de postgrado es necesario abordar el tema que nos convoca en una disciplina del conocimiento, en este caso, las ciencias militares no se contemplan en la clasificación internacionalizada de la educación en sus áreas y subáreas del conocimiento (Unesco, 1997/2006) ni en la clasificación del Manual de Frascati (OCDE, 2015).

En un esfuerzo por categorizarlas, Suárez (2005) las define como una disciplina contemporánea, que surge después de la posguerra, la cual se encuentra asociada con las ciencias de la gestión y emerge como un conjunto interrelacionado de áreas que se emplean desde sus métodos y técnicas para solucionar problemas de la realidad castrense, por ejemplo: administración militar, investigación operativa, teoría de sistemas, teoría de la decisión, planeación estratégica, entre otras. 
A su vez, Esquivel (2015) aborda las ciencias militares desde un punto de vista ontológico y realiza un análisis citando a Kuhn (2004), quien menciona que desde lo teórico "la ciencia se rige por sus propias leyes y su reconocimiento subyace a la comunidad que las practica" (p. 293), y finaliza haciendo una reflexión sobre la necesidad de reconstruir cuáles fueron los fundamentos de las ciencias militares que desarrollaron los españoles y que tuvieron adaptaciones en lo geográfico y cultural, por lo que incidieron en la evolución de ese saber.

Por otra parte, Prince define las ciencias militares "como una red transdisciplinaria, que permite predicciones fuera del rango de las disciplinas tecnológicas para hacer posible una contribución que requerirá de un análisis ético, moral y axiológico de los valores" (2008, p. 26).

Desde otra perspectiva, Ortega, citando a Kaplan (1944), indica que la ciencia militar es "el conjunto de preceptos y principios para el acertado empleo de las fuerzas militares en las operaciones bélicas y de la organización de las instituciones militares, a fin de que estas lleguen a su objetivo y éxito" (2010, p. 47).

Así, la educación militar y policial en Colombia en el siglo xxI, desde su marco normativo planteado en el Proyecto Educativo de las Fuerzas Armadas (PEFA), expone que las ciencias militares son una disciplina derivada de la rama de las ciencias sociales que estudia y entrena a los militares en ciencia o arte de la navegación aérea para el transporte a través de los aires, integrando los principios y las virtudes en la disciplina militar con conciencia aeronáutica (MINDEFENSA, 2008).

Así mismo, la FAC, a través de sus programas de pregrado define las ciencias militares como una disciplina profesional, en las que convergen campos del conocimiento tales como las ciencias humanas, sociales y básicas (Escuela Militar de Aviación, 2018).

En suma, el estudio de las ciencias militares aeronáuticas no difiere de las presunciones, métodos o premisas de los campos de conocimiento relacionados con las ciencias militares. Por lo general, estos objetos de estudio hacen énfasis sobre los aspectos prácticos y teóricos que fundamentan tanto el conocimiento militar, como las capacidades del poder aéreo para la defensa y la seguridad nacional.

Por lo tanto, para los fines que persigue este estudio, se llega al entendimiento de que las ciencias militares son multidisciplinares, interdisciplinares y transdisciplinares, contando con un fuerte componente de 
estrategia y liderazgo militar. Adicionalmente, cuando converge la educación en el aspecto aeronáutico, se envuelven las capacidades para conducir operaciones aéreas, inteligencia militar aérea, logística aeronáutica, seguridad y defensa de bases aéreas, desarrollo tecnológico, y, en general, aquellos conocimientos que fortalecen la doctrina militar aeronáutica junto con el poder aéreo y espacial nacional.

\section{Ciencias militares aeronáuticas como programa académico}

Comprendido el objeto de estudio de las ciencias militares aeronáuticas en Colombia y las consideraciones y reflexiones que diversos autores tienen sobre el tema, es importante reconocer algunos aspectos de la MACMA, en particular, su fundamentación académica. En concordancia con lo establecido en el Plan Estratégico del Sistema Educativo de las Fuerzas Armadas (PESE) (MINDEFENSA, 2007), esta se relaciona específicamente con la estrategia institucional de

consolidar la identidad educativa y garantizar la formación, actualización, capacitación, instrucción y entrenamiento más adecuados, incorporando una visión estratégica para la educación militar y policial, de tal forma que esta se convierta en el eje estructurador de la cultura institucional (MINDEFENSA, 2007, p. 11).

El Ministerio de Educación Nacional otorgó el registro calificado al programa MACMA mediante la resolución n. ${ }^{\circ} 123.827$ del 13 septiembre del 2013, el cual tiene una malla curricular que incluye componentes académicos, teóricos y prácticos orientados al estudio de la doctrina militar y a los saberes propios del quehacer militar (Documento Maestro MACMA, 2014).

Es así como la maestría contiene en su plan de estudios cuatro ejes temáticos que pretenden profundizar conocimientos y consolidar competencias propias del militar en Colombia, a saber: liderazgo, doctrina, gestión organizacional, conocimiento y empleo de los recursos militares del poder aéreo en el país.

El liderazgo como componente curricular se considera un aspecto relevante y fundamental en la formación académica de los oficiales, puesto que: 
[...] a diferencia de otras profesiones para las que el liderazgo constituye una cualidad sobresaliente en ciertos individuos, en la profesión castrense este es un imperativo para el ejercicio de la autoridad que legalmente un acto de gobierno otorga y la sociedad le reconoce a un militar (Manual de Estado Mayor, 2003).

Otro componente curricular, la toma de decisiones, constituye también un aspecto fundamental en la oferta educativa de la MACMA, ya que se considera una característica inherente de los comandantes en la estructura organizacional castrense, pues la habilidad de un comandante en este campo determina su éxito personal, laboral y por lo tanto el éxito de la institución.

Respecto a sus ejes temáticos, la MACMA guarda un rasgo distintivo - incluso si se le compara con otras ofertas académicas similares del mundo-, lo que evidencia la importancia que tiene para la Fuerza Aérea el estudio del conflicto armado y el papel de la FAC en este contexto.

Los estudios sobre derechos humanos, marcos regulatorios, convenios y tratados internacionales también componen un rasgo distintivo en el plan curricular que complementa los estudios sobre los fenómenos de la guerra y garantizan una adecuada preparación profesional para el oficial de la FAC.

Fundamentalmente, los estudios en doctrina militar constituyen el principal componente curricular del programa MACMA. La doctrina, considerada el fundamento de la filosofía castrense, contiene los principios, criterios y teorías que orientan el empleo del poder militar en el ámbito aeronáutico. Sin embargo, ese conocimiento no solo abarca teorías tradicionales, sino perspectivas de análisis sobre amenazas futuras (nuevas guerras), empleo de los recursos militares (política, estrategia), análisis de las funciones del militar en escenarios políticos futuros (posconflicto), entre otros aspectos académicos de interés.

\section{Apuntes claves de la investigación en trayectoria investigativa de las ciencias militares aeronáuticas}

\section{Recolección de la información y procesamiento de datos}

Antes de continuar, es importante aclarar cómo fueron tomados los datos que sirvieron para la realización el siguiente apartado. Esta investigación comenzó con la búsqueda de la información en los proyectos de grado de la 
MACMA desde enero del 2016 hasta julio del 2020, los cuales se encuentran de manera física y virtual en la biblioteca de la EPFAC. Después se procedió a su lectura para diligenciar unas categorías en este estudio, la cuales se recopilaron en una matriz de análisis de consistencia.

En el análisis de los trabajos de grado desarrollados durante el tiempo mencionado en el programa MACMA, se evidencia cómo ha sido la trayectoria investigativa de la Maestría en Ciencias Militares Aeronáuticas en Colombia y algunas tendencias en el campo de la investigación formativa que podrían contribuir a la toma de decisiones de los programas académicos de nivel postgradual en la FAC.

Inicialmente, debe aclararse que este trabajo define la trayectoria investigativa que describe Morín (2000) a Pérez (2012) como el desempeño o la actuación integral del sujeto, lo que implica conocimientos factuales o declarativos, habilidades, destrezas, actitudes y valores dentro de un contexto ético y científico. Este desempeño o actuación emerge de la intersección entre los conocimientos factuales y declarativos - saber conocer-, habilidades y destrezas - saber hacer- y, actitudes y valores - saber ser- (Pimienta, 2012).

En este sentido, es primordial recordar que la educación superior debe ser la formación por competencias, como una política clave para la educación (Tobón, 2008), ya que existe para solucionar problemas y situaciones.

Actualmente, gracias a las nuevas tecnologías y al empleo del poder aéreo y espacial, el estudio de las ciencias militares y aeronáuticas ha tomado fuerza y liderazgo, lo cual se refleja en la gestión académica desarrollada a través de los programas de pregrado de Ciencias Militares Aeronáuticas de la Escuela Militar de Aviación Marco Fidel Suárez y la Maestría de Ciencias Militares Aeronáuticas de la Escuela de Postgrados de la EPFAC.

Esto se aborda en la investigación de Brochero (2021), estudio en el que se analiza la definición de las ciencias militares aeronáuticas como disciplina del conocimiento; que si bien, no se encuentran normalizadas en la clasificación de las disciplinas del conocimiento de referencia global, Suárez (2005) sí las define como una disciplina contemporánea. Por esto, el diseño metodológico abordó los proyectos de grado realizados y publicados desde enero del 2016 hasta julio del 2020, mediante un proceso sistemático de recolección y análisis (Tejedor, 2000).

En el segundo apartado se presenta el estado del arte a partir de la exploración de los proyectos de grado cuya naturaleza temática son las 
ciencias militares aeronáuticas durante el tiempo indicado en el programa MACMA. Para ello, fuera de los instrumentos de análisis, Brochero (2021) consultó los estudios relacionados de Gómez, Galeano y Jaramillo (2015) y Ruiz y Bernal (2014), para así comprobar que los instrumentos empleados fueron validados (Villavicencio et al., 2016).

En el tercer apartado del análisis que adelantó Brochero (2021) se define la metodología empleada, la cual consistió en la recolección de la información con el abordaje de la muestra (57 proyectos de grado) y las matrices usadas en la recolección de datos. En referencia a la metodología utilizada para el estado del arte, cabe resaltar que, durante esta búsqueda, no se encontraron estudios dentro del campo de las ciencias militares con esta metodología.

El cuarto apartado se identificaron y analizaron los resultados obtenidos de las categorías a priori (ocho categorías), denominadas así al ser la base de los resultados de la trayectoria investigativa de las ciencias militares aeronáuticas en Colombia: objeto del estudio, palabras clave, metodología (enfoque del estudio y tipo de estudio), muestra, técnicas e instrumentos, área funcional de aplicación en la FAC, línea de investigación (eje temático y tema) y, por último, las referencias.

Del mismo modo, las subcategorías emergentes identificadas por este estudio (siete preguntas), refuerzan el aporte a la trayectoria; estas son: oficiales del cuerpo en la FAC, profesión, elección del tema, número de asesores temáticos, número de asesores metodológicos, aporte a la FAC e implementación.

Finalmente, el presente estudio evidenció la trayectoria investigativa de las ciencias militares aeronáuticas generada de los proyectos de grado de la maestría en su estructura, objetos de estudio y metodología desarrolladas.

La mayoría de los estudiantes de la maestría adelantaron temas de investigación alineados con la educación y el desarrollo de las capacidades de la FAC. Asimismo, realizaron trabajos sobre el Comando de Operaciones Aéreas (COA), el Comando de Personal (COP), la Jefatura de Educación Aeronáutica y el Control de Eventos de Seguridad Operacional (EVESOS).

Con respecto a los aspectos operativos, se encontró que la mayoría de los egresados de la maestría son de la Escuela Militar de Aviación (EMAVI) y del programa de Administración Aeronáutica.

De modo que el estudio de Brochero (2021) es importante porque logra identificar las categorías a priori y emergentes que orientan la investigación 
en el área de las ciencias militares aeronáuticas en Colombia: estructura de trabajo de grado, tema de investigación, aspectos operativos y aportes de la investigación.

\section{Categoría 1. Estructura del proyecto de grado}

Tiene asociada la categoría objeto del estudio, en la cual se encontró que el 14 \% trata sobre temas de educación, capacitación en la FAC y en EMAVI. A su vez, después de procesar la categoría palabras clave en la que se encontraron 243, se agruparon las siguientes: militar y aéreo, que representan el 4,3\% y el 3,8 \% respectivamente. Se destacan otras como Vehículos Aéreos no Tripulados (UAV), tripulado, capacidad y drones.

Asimismo, fue distintiva la categoría enfoque metodológico y el tipo de investigación cualitativa, que figuran con el $61 \%$ de los proyectos de grado, de los cuales, el 51, $4 \%$ son cualitativos-descriptivos. Con relación a la categoría muestra, el 83 \% de los proyectos de grado se realizó con sujetos y el 7 \% consistió en la revisión de EvEsos; esto se relaciona con la categoría medios empleados en la recopilación de los datos, ya que la muestra corresponde en un $34 \%$ a recuperación documental y en un 37 \% a la realización de encuestas y entrevistas. Finalmente, en la categoría número de referencias el $57 \%$ de los proyectos de grado contienen entre el $13 \%$ y $50 \%$.

\section{Categoría 2. Tema de investigación}

Para esta se asocian las categorías emergentes que surgieron de la matriz analítica, de la cual se obtuvo el siguiente análisis: la mayoría de los trabajos de grado están dirigidos al COA con un 31 \% de representatividad, seguido por el Cop. En correspondencia con las líneas de investigación de la MACMA, el $64 \%$ de los trabajos se ubican en la línea doctrina militar aeronáutica, el $22 \%$ selecciona el eje temático toma de decisiones y el $36 \%$ empleo y desarrollo del poder aéreo. Es importante aclarar que en el eje temático pensamiento político-militar no se evidencian investigaciones asociadas. Para la selección del tema del proyecto de grado, el 18 \% de los estudiantes lo eligieron porque tenían conocimientos al respecto. 


\section{Categoría 3. Aspectos operativos de la opción de grado}

Para esta categoría se encontró que el perfil de los oficiales que ingresan a la maestría corresponde en un 71\% a oficiales egresados de la Escuela Militar de Aviación. En cuanto a los programas de pregrado el más cursado es el de Administración Aeronáutica y algunos estudiantes son pilotos, quienes representan el $54 \%$ de las profesiones.

\section{Categoría 4. Aportes de la investigación}

Para esta categoría se encontró que el 97 \% de los egresados que respondieron el cuestionario manifestó que su proyecto de grado sí aportó a la FAC. El 41 \% indicó que sus proyectos están siendo implementados en la FAC o sirven para la ejecución de proyectos, manuales o toma de decisiones. El 31 \% de los trabajos solucionó un problema en COA y un 30 \% fueron aplicados al cop. En cuanto al área de conocimiento el, $64 \%$ de los trabajos de grado respondieron a los pretextos investigativos de la línea de doctrina militar aeronáutica en la cual un $22 \%$ abordaron temas relacionados con la toma de decisiones y un 36 \% respondió a lo indicado en la línea de investigación empleo y desarrollo del poder aéreo.

\section{Reflexión final}

En síntesis, la trayectoria investigativa de las ciencias militares aeronáuticas en Colombia comenzó con la creación de la maestría en el 2014, y a partir del 2019 se observa un crecimiento de proyectos de grado que aportan significativamente al desarrollo investigativo, evidenciando el surgimiento de tendencias de investigación relacionadas con tópicos como UAV y drones, que crean las líneas de conocimiento y nuevos temas de investigación. Igualmente, se destaca el aporte de investigación desde la MACMA a la FAC, porque en su mayoría resuelven problemas de investigación de sus áreas funcionales; es así como esta información muestra las tendencias investigativas de los egresados, para la generación de núcleos de investigación y redes de conocimiento para futuros estudiantes.

Por otra parte, la información presentada en el estudio de Brochero (2021) constituye un insumo para los programas académicos con enfoque en ciencias militares aeronáuticas, el cual permite a docentes y estudiantes 
conocer las investigaciones que están surgiendo en este campo de conocimiento desde la consecución y síntesis de los datos de los proyectos investigativos generados en la MACMA. Lo anterior posibilita conocer el estatus de la maestría en los últimos años y dar un insumo a las directivas para trazar el horizonte investigativo con líneas de investigación y núcleos temáticos según las demandas del área de conocimiento.

\section{Conclusiones}

En resumen, se infiere que las ciencias militares actúan en la organización y labor de las fuerzas militares en el proceso de gestión, análisis y evolución de los conflictos. Además de realizar actividades que prevengan conflictos armados o la articulación de procesos que conlleven a generar estabilidad política y social.

La trayectoria investigativa en la MACMA incluye temas inherentes a la ciencia y la tecnología. Asimismo, aborda algunos de los métodos de investigación cualitativa con un enfoque marcado en la doctrina militar en referencia a conceptos claves de rigor y evaluación académica.

Es así como los trabajos de grado revisados describen los métodos empleados y las características principales de la investigación cualitativa, en los cuales se abordan los principales temas y preocupaciones de quienes se dedican a la profesión militar en el ámbito aeronáutico.

\section{Referencias}

Brochero, D.P. (2021). Trayectoria investigativa de las Ciencias militares aeronáuticas en Colombia durante el periodo 2016-202o [tesis de maestría, Escuela de Postgrado Fuerza Aérea Colombiana]. Sistema de bibliotecas Fuerza Pública Colombiana.

Congreso de Colombia. (1992, 28 de diciembre). Fundamentos de la Educación Superior. [Ley 30 de 1992]. https://www.mineducacion.gov.co/1621/articles-86437_ Archivo_pdf.pdf

Escuela de Postgrados Fuerza Aérea Colombiana. (2018). Informe de autoevaluación con fines de renovación de registro calificado Maestría En Ciencias Militares Aeronáuticas. Escuela de Postgrados de la Fuerza Aérea Colombiana. https://www. epfac.edu.co/sites/epfac/files/Docs-EPFAC/normatividad/Sistema\%20Insti tucional\%20de\%20Aseguramiento\%20de\%20la\%20Calidad/sistema_institu cional_de_aseguramiento_de_la_calidad.pdf 
Escuela de Postgrados Fuerza Aérea Colombiana. (2019). Documento maestro ciencias militares aeronáuticas. Escuela de Postgrados de la Fuerza Aérea Colombiana.

Escuela de Postgrados Fuerza Aérea Colombiana. (2019). Reglamento Académico. Secretaria Académica. Escuela de Postgrados de la Fuerza Aérea Colombiana. https://cdn227724.fac.mil.co/sites/epfac/files/Docs-EPFAC/normatividad/re glamento\%20acad\%C3\%A9mico\%202019/reglamento_academico_y_discipli nario_6_de_sep_de_2019.pdf

Escuela de Postgrados Fuerza Aérea Colombiana. (2021). Lineamientos para el desarrollo de la Investigación Formativa EPFAC. ESINV. Escuela de Postgrados de la Fuerza Aérea Colombiana. https://www.epfac.edu.co/sites/epfac/files/Docs-EPFAC/normati vidad/Lineamientos\%20para\%20la\%20Articulaci\%C3\%B3n\%20de\%20la\%20 Investigaci\%C3\%B3n/Lineamientos\%20para\%20la\%20articulacion\%20Inves tigacion\%202021.pdf

Esquivel, R. (2015). Modernidad hispana en las ciencias militares en Colombia. Revista Científica General José María Córdova, 13(15), 291-307. https://bit.ly/3qEjgE2

Escuela Militar de Aviación. (2018). Proyecto Educativo del Programa de Maestría de Ciencias militares aeronáuticas. Escuela Militar de Aviación. https://www.emavi. edu.co/sites/emavi/files/imagenesemavi/documentos/Proyecto\%20Educa tivo\%20Programa\%20Ciencias\%20Militares\%20Aerona\%C3\%BAticas\%20 (PEP\%20PCMAE)\%202020.pdf

Gómez, M., Galeano, C., \& Jaramillo, D. (2015). El estado del arte: una metodología de investigación. Revista Colombiana de Ciencias Sociales, 6(2), 423-442. http://bit. ly/2MfI1ra

Kuhn, T. (2004). La estructura de las revoluciones científicas. Fondo de Cultura Económica. https://materiainvestigacion.files.wordpress.com/2016/05/kuhn1971.pdf

Ministerio de Defensa Nacional. (2008). Proyecto Educativo de las Fuerzas Armadas (PEFA). Biblioteca Escuela Militar de Cadetes General José María Córdova.

Ministerio de Defensa Nacional. (2007). Plan Estratégico del Sistema Educativo de las Fuerzas Armadas (PESE) 2007-2019. https://bit.ly/3sXDXws

Organización para la Cooperación y el Desarrollo Económico. (2015). Frascati Manual 2015: Guidelines for Collecting and Reporting Data on Research and Experimental Development. The Measurement of Scientific, Technological and Innovation Activities, OECD Publishing. https://www.oecd.org/sti/frascati-ma nual-2015-9789264239012-en.htm

Ortega, R. (2010). Ciencias Militares. Ciencia-arte método-estado mayor. Ejército de Chile; Academia de Guerra; Jefatura de Estudios. http://bit.ly/2KOTptO

Ministerio de Educación Nacional. (2013). Resolución 12.382 del 2013.

Pimienta, J. (2012). Las competencias en la docencia universitaria. Pearson. https:// www.academia.edu/33825697/Las_competencias_en_la_docencia_univer sitaria_pimienta_1_ 
Presidencia de la República de Colombia. (2019, 25 de julio). Decreto 1330 de julio del 2019. https://www.mineducacion.gov.co/portal/normativa/Decretos/387348: Decreto-1330-de-julio-25-de-2019

Prince, S. (2008). Los fundamentos de las ciencias militares. Revista Política y Estrategia, (111), 14-28. http://bit.ly/3oafeBG

Ruiz, H., \& Bernal, Y. (2014). Estado del arte de los trabajos de grado realizados en el programa de licenciatura en educación básica con énfasis en humanidades e idiomas de la Facultad de Ciencias de la Educación de la Universidad Libre desde el primer semestre de 2009 al primer semestre de 2013 [tesis de pregrado, Universidad Libre]. https:// bit.ly/39T75wi

Sabino, C. (1986). El proceso de investigación. Panapo.

Sistema Nacional de Información de la Educación Superior. (2021). Qué es el SNIES. Ministerio de Educación Nacional. https://snies.mineducacion.gov.co/portal/ EL-SNIES/Que-es-el-SNIES/

Suárez, J. (2005). ¿Qué son las Ciencias Militares? Revista Científica General José María Córdova, 3(3), 45-48. https://bit.ly/30aUvxu

Tejedor, J. (2000). El diseño y los diseños en la evaluación de programas. Revista de Investigación Educativa, 18(2), 319-339. https://bit.ly/3qIKxVO

Tobón, S. (2008). La formación basada en competencias en la educación superior: El enfoque complejo. Universidad Autónoma de Guadalajara. https://www.researchgate. net/publication/329440312_La_formacion_basada_en_competencias_en_la_ educacion_superior_el_enfoque_complejo

Villavicencio, E., Ruiz, E., \& Cabrera, A. (2016). Validación de cuestionarios. Revista OACTIVA, 1(3), 75-80. https://bit.ly/3CF4JAx 